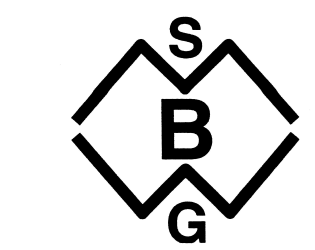

BELGIAN MATHEMATICAL

SOCIETY

Reprint from the Bulletin of the Belgian Mathematical Society - Simon Stevin

\title{
Regular methods of summability and the weak $\sigma$-Fatou property in abstract Banach lattices of integrable functions
}

\author{
E. Jiménez Fernández $\quad$ M. A. Juan \\ Enrique A. Sánchez Pérez
}

Bull. Belg. Math. Soc. Simon Stevin 25 (2018), 545-553

The Bulletin of the Belgian Mathematical Society - Simon Stevin is published by The Belgian Mathematical Society, with financial support from the Universitaire Stichting van Belgie - Fondation Universitaire de Belgique and the Fonds National de la Recherche Scientifique (FNRS). It appears quarterly and is indexed and/or abstracted in Current Contents, Current Mathematical Publications, Mathematical Reviews, Science Citation Index Expanded and Zentralblatt für Mathematik.

The Bulletin of the Belgian Mathematical Society - Simon Stevin is part of Project Euclid (Cornell University Library), an aggregation of electronic journals. It is available online to subscribers to Project Euclid (http:/ / projecteuclid.org).

For more informations about the Belgian Mathematical Society - Simon Stevin, see our web site at http://bms.ulb.ac.be 



\title{
Regular methods of summability and the weak $\sigma$-Fatou property in abstract Banach lattices of integrable functions
}

\author{
E. Jiménez Fernández M. A. Juan \\ Enrique A. Sánchez Pérez
}

\begin{abstract}
Consider an abstract Banach lattice. Under some mild assumptions, it can be identified with a Banach ideal of integrable functions with respect to a (non necessarily $\sigma$-finite) vector measure on a $\delta$-ring. Extending some nowadays well-known results for the Komlós property involving Cesaro sums, we prove that the weak $\sigma$-Fatou property for a Banach lattice of integrable functions $E$ is equivalent to the existence for each norm bounded sequence $\left(f_{n}\right)$ in $E$ of a regular method of summability $D$ such that the sequence $\left(f_{n}^{D}\right)$ converges.
\end{abstract}

\section{Introduction}

The relevant Komlós Theorem on the hereditary Cesaro summability of a subsequence of any bounded sequence in $L^{1}[0,1]$ is nowadays known to be also true in Banach ideals of integrable functions whenever they satisfy the weak $\sigma$-Fatou property. It was shown by Day and Lennard in 2010 [6]. These results are however strongly dependent on the fact that the spaces are defined over an integration structure that is constructed having a $\sigma$-finite measure as a starting point. Although this situation covers a lot of spaces, there are still some relevant

Received by the editors in November 2017 - In revised form in February 2018.

Communicated by F. Bastin.

2010 Mathematics Subject Classification : Primary 40C99; Secondary 28A20, 46G10, 46E30.

Key words and phrases : Banach lattice, Fatou property, regular methods of summability, integrable functions, vector measure. 
Banach lattices that cannot be described using this framework - for example $\ell_{p}(I)$-spaces for a non countable set of indexes $I, 1 \leq p \leq \infty$ - In [12], the authors of the present paper showed how far this equivalence can be extended when general Banach lattices are considered. The main tool for doing this was the recently developed representation theory for abstract Banach lattices using spaces of $v$-integrable and weakly $v$-integrable functions with respect to a vector measure $v$ defined on a $\delta$-ring (see $[4,7,8]$; see also [15, Ch.3] and the references therein for the finite measure case). This allows, for instance, to include lattices as the $\ell_{p}(I)$-spaces mentioned above.

The aim of this note is to show that the main result that was proved in [12] can be extended for giving this characterization by means of a Komlós type property but changing the hereditary Cesaro summability of a subsequence through hereditary summability by any other regular summability method. We prove the following result, that in a sense closes the analysis of the equivalence among regular summability properties of bounded sequences and the weak $\sigma$-Fatou property, and establishes the limits for this to hold in terms of the measure space. Note that no $\sigma$-finiteness of the measure is needed, and that the result can be applied to any Banach lattice which allows to be represented as a space of integrable functions; for instance, under some mild requirements any order continuous Banach lattice can be written as such an space. Let $v$ be a vector measure on a $\delta$-ring $\mathcal{R}$ and write $\mathcal{R}^{\text {loc }}$ for the $\sigma$-algebra associated by $\mathcal{R}$. Let $L^{1}(v)$ be the Banach lattice of all (classes of $v$-a.e. equal) $v$-integrable functions.

Theorem 1.1. Let E be a Banach lattice of classes of v-a.e. equal $\mathcal{R}^{\text {loc }}$-measurable functions which is also an ideal of $L^{1}(v)$. The following statements are equivalent.

(i) E has the weak $\sigma$-Fatou property.

(ii) E has the Komlós property.

(iii) For each norm bounded sequence $\left(f_{n}\right)_{n} \subset E$ there is a regular method of summability $D$ such that $\left(f_{n}^{D}\right)_{n}$ converges $v$-a.e. to a measurable function $f^{D}$ in $E$.

Here $f_{n}^{D}$ is written for the $n$-th sum resulting of the application of $D$ to the sequence $\left(f_{n}\right)_{n}$. Let us introduce some concepts and notation regarding summability. Let $D=\left(d_{n, m}\right)$ be an infinite matrix of scalars and let $\left(x_{n}\right)_{n}$ be a convergent sequence in a Banach space $X$. We will say that $D$ defines a regular method of summability, if for each $n \in \mathbb{N}$, the sequence $x_{n}^{D}=\sum_{m=1}^{\infty} d_{n, m} x_{m}$ exists and is convergent to the same limit as $\left(x_{n}\right)_{n}$. The following result will be crucial for us (see [9], Theorem 1). A scalar infinite matrix $D=\left(d_{n, m}\right)$ is a regular method of summability if and only if it satisfies the following three conditions that do not depend on the Banach space $X$.

1. $\sum_{m=1}^{\infty}\left|d_{n, m}\right| \leq M$ for some $M>0$ and for all $n \in \mathbb{N}$.

2. $\lim _{n} d_{n, m}=0$ for all $m \in \mathbb{N}$.

3. $\lim _{n} \sum_{m=1}^{\infty} d_{n, m}=1$. 
Notice that if we drop an infinite (or finite) number of rows or if we add an infinite (or finite) number of columns of zeroes in a regular method of summability, we still have a regular method of summability.

A relevant result regarding regular methods of summability is due to Erdös and Magidor (see p.2 in [11]). It establishes that for a given bounded sequence of a Banach space, there is a subsequence such that two mutually exclusive options are given: either it has a subsequence such that each subsequence of it converges with respect to $D$ (same limit), or there are no subsequences that converge respect to $D$. This fact will be implicitly used in our arguments for proving Theorem 1.1.

A particular method of summability is the one related to the Cesaro sums which involves the so called Komlós property of a Banach function space $X(\mu)$, as follows. Let $\left(f_{n}\right)_{n}$ be a bounded sequence in $X(\mu)$, then there exists a subsequence $\left(f_{n_{k}}\right)_{k}$ and a function $f \in X(\mu)$ such that for any further subsequence $\left(h_{j}\right)_{j}$ of $\left(f_{n_{k}}\right)_{k}$, the series $\frac{1}{n} \sum_{j=1}^{n} h_{j}$ converges $\mu$-a.e. to $f$.

To finish this section, let us introduce some definitions and basic results that are needed on Banach lattices and spaces of integrable functions with respect to a vector measure on a $\delta$-ring.

Let $E$ be a Banach lattice with norm $\|\cdot\|$ and order $\leq$. An ideal of $E$ is a subspace $F$ in $E$ such that $y \in F$ whenever $y \in E$ with $|y| \leq|x|$ for some $x \in F$. An ideal $F$ in $E$ is said to be order dense in $E$ if for every $0 \leq x \in E$ there exists an upwards directed system $\left(x_{\tau}\right)_{\tau} \subset F$ such that $0 \leq x_{\tau} \uparrow x=\sup x_{\tau}$. The Banach lattice $E$ is said to have $\sigma$-order continuous norm, or briefly, to be $\sigma$-order continuous if for every order decreasing sequence $\left(x_{n}\right)_{n} \downarrow 0$ it follows that $\left\|x_{n}\right\| \downarrow 0$. If this property holds by means of downwards directed systems, $E$ is said to be order continuous. We denote by $E_{a n}$ the order continuous part of $E$, that is, the largest order continuous ideal in $E$. The Banach lattice $E$ satisfies the $\sigma$-Fatou property if for every norm bounded and increasing sequence $\left(x_{n}\right)_{n}$ of positive elements in $E$ we have that $x=\sup x_{n}$ exists (in $E$ ) and $\left\|x_{n}\right\| \uparrow\|x\|$. The space is said to have the weak $\sigma$-Fatou property if the last condition on the norms is not required. Again, if we consider upwards directed systems in the last definitions, we will have the Fatou and the weak Fatou properties, respectively. Finally, every bijective operator $T \in L(E, F)$ between Banach lattices which preserves the lattice operations (that is, $T(x \vee y)=T x \vee T y$ and $T(x \wedge y)=T x \wedge T y$ for all $x, y \in E$ ) is called an order isomorphism. We refer the reader to $[1,14,19,20]$ for issues related to Banach lattices.

Recall now the basis of the theory on spaces of integrable functions with respect to vector measures on a $\delta$-ring. Throughout this paper, $\mathcal{R}$ will be a $\delta$-ring of subsets of a set $\Omega$, that is, a ring of sets closed under countable intersections. We consider $\mathcal{R}^{l o c}$ the associated $\sigma$-algebra to $\mathcal{R}$ defined by $\mathcal{R}^{l o c}=\{B \subset \Omega$ : $B \cap A \in \mathcal{R}$ for every $A \in \mathcal{R}\}$ and denote by $\mathcal{M}\left(\mathcal{R}^{\text {loc }}\right)$ the space of measurable real functions on $\left(\Omega, \mathcal{R}^{\text {loc }}\right)$. Also, we use $\mathcal{S}(\mathcal{R})$ for the set of simple functions with support in $\mathcal{R}^{l o c}$. Let $v: \mathcal{R} \rightarrow X$ be a Banach valued countably additive vector measure on the $\delta$-ring $\mathcal{R}$, that is, $v\left(\cup_{n=1}^{\infty} A_{n}\right)=\sum_{n} v\left(A_{n}\right)$ in the norm topology of $X$ for every sequence $\left(A_{n}\right)_{n}$ of pairwise disjoint sets in $\mathcal{R}$ with $\cup A_{n} \in \mathcal{R}$. For each element $x^{\prime} \in X^{\prime}$ the formula $v_{x^{\prime}}(A):=\left\langle v, x^{\prime}\right\rangle(A), A \in \mathcal{R}$, defines a scalar measure (see [7, Preliminaries]), where $X^{\prime}$ denotes, as usual, the topological dual 
of $X$. We write $\left|v_{x^{\prime}}\right|$ for its variation, that is, for every $A \in \mathcal{R}^{l o c}$,

$$
\left|v_{x^{\prime}}\right|(A):=\sup \left\{\sum\left|v_{x^{\prime}}\left(A_{i}\right)\right|:\left(A_{i}\right)_{i} \text { finite disjoint sequence in } \mathcal{R} \cap 2^{A}\right\},
$$

and define the semivariation of $v$ by $\|v\|(A):=\sup \left\{\left\langle v, x^{\prime}\right\rangle(A), x^{\prime} \in X^{\prime}\right.$, $\left.\left\|x^{\prime}\right\|=1\right\}, A \in \mathcal{R}^{l o c}$. It is monotone, increasing, subadditive, finite on $\mathcal{R}$ and satisfies that

$$
\frac{\|v\|(A)}{2} \leq \sup \left\{\|v(B)\|: B \in \mathcal{R} \cap 2^{A}\right\} \leq\|v\|(A),
$$

for all $A \in \mathcal{R}^{\text {loc }}$. We say that a set $B \in \mathcal{R}^{\text {loc }}$ is $v$-null if $\|v\|(B)=0$ and that a property holds $v$-almost everywhere (briefly $v$-a.e.) if it holds except on a $v$-null set. From [3, Theorem 3.2], there always exists a measure $\lambda: \mathcal{R} \rightarrow[0, \infty]$ with the same null sets as $v$. We will refer to such kind of measure as a local control measure of $v$.

A function $f \in \mathcal{M}\left(\mathcal{R}^{\text {loc }}\right)$ is integrable with respect to $v$ (or $v$-integrable) if it is integrable for each scalar measure $\left|v_{x^{\prime}}\right|$ and moreover for every $A \in \mathcal{R}^{\text {loc }}$ there is a vector in $X$ denoted by $\int_{A} f d v$ such that for every $x^{\prime} \in X^{\prime},\left\langle\int_{A} f d v, x^{\prime}\right\rangle=\int_{A} f d v_{x^{\prime}}$. The space $L^{1}(v)$ of $v$-a.e. equal $v$-integrable functions is an order continuous Banach lattice when endowed with the norm $\|f\|_{v}:=\sup _{x^{\prime} \in B_{X^{\prime}}} \int|f| d\left|\left\langle v, x^{\prime}\right\rangle\right|$, $f \in L^{1}(v)$ and the $v$-a.e. order, containing $\mathcal{S}(\mathcal{R})$ as a dense subset both in order and in norm (see [13, Theorem 3.3]). Here $B_{X^{\prime}}$ denotes the unit ball of $X^{\prime}$. Furthermore, the space $L^{1}(v)$ is also an ideal of measurable functions, that is, if $|f| \leq|g|$ $v$-a.e with $f \in \mathcal{M}\left(\mathcal{R}^{l o c}\right)$ and $g \in L^{1}(v)$, then $f \in L^{1}(v)$.

We say that a function $f \in \mathcal{M}\left(\mathcal{R}^{\text {loc }}\right)$ is weakly $v$-integrable if it is integrable with respect to $\left|v_{x^{\prime}}\right|$ for all $x^{\prime} \in X^{\prime}$. We denote by $L_{w}^{1}(v)$ the space of functions in $\mathcal{M}\left(\mathcal{R}^{\text {loc }}\right)$ where functions which are equal $v$-a.e. are identified. This space is a Banach lattice when endowed with the norm $\|\cdot\|_{v}$ and the $v$-a.e. order in which convergence in norm of a sequence implies $v$-a.e. convergence of some subsequence (see Lemma 3.13 in [17] or Theorem 100.6 in [20]). The space $L_{w}^{1}(v)$ has always the $\sigma$-Fatou property and $L^{1}(v)$ is a closed ideal of $L_{w}^{1}(v)$. Moreover, following Theorems 3.2 and 4.2 in [4] the order continuous part of $L_{w}^{1}(v)$ is actually $L^{1}(v)\left(\left(L_{w}^{1}(v)\right)_{a n}=L^{1}(v)\right)$ and $L^{1}(v)$ is order dense in $L_{w}^{1}(v)$. Again $L_{w}^{1}(v)$ is an ideal of measurable functions.

Finally, let us recall the representation theorems of abstract Banach lattices involving spaces of integrable functions. Every order continuous Banach lattice can always be represented by means of an order isomorphism which is also an isometry by a space $L^{1}(v)$ of $v$-integrable functions with respect to a vector measure on a $\delta$-ring (see [5, pp.22-23], [8, Theorem 5]). Also, every Banach lattice $E$ with the Fatou property and having its order continuous part $E_{a n}$ as an order dense subset is order isomorphic and isometric to a space $L_{w}^{1}(v)$ of weakly $v$-integrable functions for a certain vector measure $v$ defined on a $\delta$-ring (see Theorem 10 in [8]).

For these and other issues related to spaces of integrable functions with respect to vector measures over a $\delta$-ring, see for instance $[4,7,13,16,17,18]$. 


\section{Spaces of vector measure integrable functions and regular methods of summability}

Let $v: \mathcal{R} \rightarrow X$ be a vector measure on the $\delta$-ring $\mathcal{R}$ that takes values in a Banach space $X$. We start by considering ideals of the space of integrable functions $L^{1}(v)$. The equivalence between the weak $\sigma$-Fatou property, the Komlós property (redefined in the context of $L^{1}(v)$ with the $v$-a.e. order) and the existence of summable subsequences with respect to regular methods of summability is showed in Theorem 1.1. Let us present the proof.

Proof. (Proof of Theorem 1.1)

The equivalence of $(i)$ and (ii) is done by Theorem 3.2 in [12].

Let us show now $(i i) \Rightarrow($ iii). For this aim, take a norm bounded sequence $\left(f_{n}\right)_{n} \subset E$. Clearly, by the Komlós property, there exists a subsequence $\left(f_{n_{k}}\right)_{k}$ of $\left(f_{n}\right)_{n}$ and a function $f \in E$ such that $\left(\frac{1}{n} \sum_{k=1}^{n} f_{n_{k}}\right)_{n}$ converges $v$-a.e. to $f$. Write $\frac{1}{n} \sum_{k=1}^{n} f_{n_{k}}=\sum_{k=1}^{n} \frac{1}{n} f_{n_{k}}=\sum_{k=1}^{n} c_{n, n_{k}} f_{n_{k}}$ and for each $n$, define $c_{n, m}^{\prime}=$ $c_{n, n_{k}}=\frac{1}{n}$ if $m=n_{k}, k=1, \ldots, n$, and 0 otherwise. Note that, fixing $n \in \mathbb{N}$, $\sum_{m=1}^{\infty} c_{n, m}^{\prime}=\sum_{k=1}^{n} c_{n, n_{k}}^{\prime}=\sum_{k=1}^{n} \frac{1}{n}=1$. Then there is a regular method of summability

$C^{\prime}=\left(c_{n, m}^{\prime}\right)$ such that $\left(f_{n}^{C^{\prime}}\right)_{n}$ converges $v$-a.e. to $f=f^{C^{\prime}}$ in $E$.

Finally, we prove $(\mathrm{iii}) \Rightarrow(i)$. Suppose that $E$ does not have the weak $\sigma$-Fatou property. Then, there exists an increasing positive sequence $0 \leq f_{n} \uparrow \sup _{n} f_{n}=$ $f \in \mathcal{M}\left(\mathcal{R}^{\text {loc }}\right)$ (that is $v$-a.e.) with $\sup _{n}\left\|f_{n}\right\|_{v}<+\infty$ such that $f \notin E$. Taking into account that for every $n, f_{n}$ is also in $L^{1}(v)$, there exists a sequence $\left(\varphi_{n, k}\right)_{k} \subset \mathcal{S}(\mathcal{R})$ so that $\varphi_{n, k} \uparrow f_{n}$, that is, for every $n,\left(\varphi_{n, k}\right)_{k}$ converges $v$-a.e. to $f_{n}$.

Consider the set union $\bigcup_{n} \operatorname{supp}\left(f_{n}\right)$ of the supports of the functions involved and notice that $\operatorname{supp}\left(f_{n}\right) \subset \bigcup_{k} \operatorname{supp}\left(\varphi_{n, k}\right)$ for every $n$, then $\bigcup_{n} \operatorname{supp}\left(f_{n}\right)$ can be covered $v$-a.e. by a sequence $\left(A_{m}\right)_{m} \subset \mathcal{R}$. Denote $U:=\bigcup_{m} A_{m}$ and note that we can write $U$ as a disjoint union of countably many sets of $\mathcal{R}$ of positive semivariation just taking the sets $B_{k}:=A_{k} \backslash \bigcup_{n=k+1}^{\infty}\left(A_{n} \cap A_{k}\right), k=1,2, \cdots$ and dropping the empty sets. Clearly, the $v$-a.e. convergence of $\left(f_{n}\right)_{n}$ can be considered in $U$ by rejecting the corresponding $v$-null set. Moreover, following 2.1 in [12], the projection band $P_{L_{w}^{1}(v)}(U)$ defined on $L_{w}^{1}(v)$ by the characteristic function $\chi_{U}$ and the space $L_{w}^{1}\left(\left.v\right|_{U}\right)$ are isometric and order isomorphic, where $v_{U}:=v_{\mathcal{R} \cap U}$ and so, the same occurs with the projection band $P_{L_{w}^{1}(v)}(U)$ in $E$ (that will be denoted by $P_{E}(U)$ ). Consequently, $v$-a.e. convergence is equivalent to $v_{U}$-a.e. convergence for $\left(f_{n}\right)_{n}$. Then, we actually have a sequence $\left(f_{n}\right)_{n} \subset P_{E}(U)$ converging to $f$ $v_{U}$-a.e. and so that $\sup _{n}\left\|f_{n}\right\|_{v}<+\infty$ where $f \notin P_{E}(U)$. Remark that $f \in P_{L_{w}^{1}(v)}(U)$ due to the $\sigma$-Fatou property of $L_{w}^{1}(v)$.

Take now the $\delta$-ring $\mathcal{R} \cap U \subset \mathcal{R}$ (for the proof of this fact just take into account that for every $\left.B \in \mathcal{R}, B \cap U=B \cap\left(\bigcup_{n} B \backslash B_{n}\right)=B \backslash\left(\cap_{n} B \backslash B_{n}\right) \in \mathcal{R}\right)$. Since the vector measure $v_{U}$ is $\sigma$-finite over $\mathcal{R} \cap U$, it is possible to construct a bounded (local) control measure for $v_{U}$ as follows (see a similar construction in Theorem 3.3 in [7]). For every $n$, consider $\left.v\right|_{\mathcal{R} \cap B_{n}}$ which is a vector measure defined on a $\sigma$-algebra. Then take a Rybakov control measure $\mu_{n}=\left.v_{x^{\prime}}\right|_{\mathcal{R} \cap B_{n}}$ for $\left.v\right|_{\mathcal{R} \cap B_{n}}$ (see Chapter 9.2 in [10] for the existence of such a measure) and define 
$\mu_{U}: \mathcal{R} \cap U \rightarrow\left[0,+\infty\left[\right.\right.$ by $\mu_{U}(A):=\sum_{n} \frac{\mu_{n}\left(A \cap B_{n}\right)}{2^{n} \max \left\{1, \mu_{n}\left(B_{n}\right)\right\}}$. Clearly $\mu_{U}$ is a finite countably additive measure on the $\delta$-ring (and also a semi-ring) $\mathcal{R} \cap U$ which is equivalent to $v_{U}$.

Classical arguments allows us to extend $\mu_{U}$ to $\mathcal{R}^{\text {loc }} \cap U=(\mathcal{R} \cap U)^{\text {loc }}$. Let us show this fact in detail. First, remark that $\mathcal{R}^{l o c} \cap U=(\mathcal{R} \cap U)^{l o c}$ as $\sigma$-algebras of subsets of $U$. For the inclusion $\mathcal{R}^{l o c} \cap U \subset(\mathcal{R} \cap U)^{l o c}$, fix $A \in \mathcal{R}^{l o c}$ with $A \subset U$ and $B=C \cap U \in \mathcal{R} \cap U$, then $A \cap B=A \cap C \cap U \in \mathcal{R} \cap U$ due to the definition of $\mathcal{R}^{\text {loc }}$ and the inclusion is proved. For the converse, fix $A \in(\mathcal{R} \cap U)^{l o c}$ with $A \subset U$ and $C \in \mathcal{R}$. Hence $A \cup C \in \mathcal{R} \cap U$ due to the definition of $(\mathcal{R} \cap U)^{l o c}$. The relation $\mathcal{R} \cap U \subset R$ gives the converse inclusion. On the other hand, using the Carathéodory extension procedure (see for example Section 10 in [2]), we get that $\mu_{U}$ can be extended to the $\sigma$-algebra $\sum_{\mu_{U}}$ of measurable sets with respect to the outer measure defined by $\mu_{U}^{*}$. Consequently, it suffices to show that each set in $(\mathcal{R} \cap U)^{l o c}$ is actually in $\sum_{\mu_{U}}$. For this aim and following for instance Lemma 9.26 in [2], we have to prove that for a fixed set $B \in(\mathcal{R} \cap U)^{l o c}, \mu_{U}(A)=\mu_{U}^{*}(A \cap B)+\mu_{U}^{*}\left(A \cap B^{C}\right)$ for each $A \in \mathcal{R} \cap U$. Clearly, $A \cap B$ and $A \cap B^{C}$ are members of $\mathcal{R} \cap U$ due to the definition of $\mathcal{R}^{l o c}$, and so $\mu_{U}^{*}(A \cap B)=\mu_{U}(A \cap B)$ and $\mu_{U}^{*}\left(A \cap B^{C}\right)=\mu_{U}\left(A \cap B^{C}\right)$. Hence, we really have to check that

$$
\sum_{n} \frac{\mu_{n}\left(A \cap B_{n}\right)}{2^{n} \max \left\{1, \mu_{n}\left(B_{n}\right)\right\}}=\sum_{n} \frac{\mu_{n}\left(A \cap B \cap B_{n}\right)}{2^{n} \max \left\{1, \mu_{n}\left(B_{n}\right)\right\}}+\sum_{n} \frac{\mu_{n}\left(A \cap B^{C} \cap B_{n}\right)}{2^{n} \max \left\{1, \mu_{n}\left(B_{n}\right)\right\}},
$$

for every $A \in \mathcal{R} \cap \mathcal{U}$. The identity $\mu_{n}\left(A \cap B_{n}\right)=\mu_{n}\left(A \cap B \cap B_{n}\right)+$ $\mu_{n}\left(A \cap B^{C} \cap B_{n}\right)$ gives the result.

We use again $\mu_{U}$ for the extension. We claim now that $f \in L^{1}\left(\mu_{U}\right)$. In fact,

$$
\begin{aligned}
\int f d \mu_{U} & =\sum_{n} \frac{1}{2^{n}} \int_{B_{n}} f \frac{d \mu_{n}}{\max \left\{1, \mu_{n}\left(B_{n}\right)\right\}} \leq \sup _{n} \int_{B_{n}} f \frac{d \mu_{n}}{\max \left\{1, \mu_{n}\left(B_{n}\right)\right\}} \\
& =\sup _{n} \frac{1}{\max \left\{1, \mu_{n}\left(B_{n}\right)\right\}} \int_{B_{n}} f d \mu_{n}=\left.\sup _{n} \frac{1}{\max \left\{1, \mu_{n}\left(B_{n}\right)\right\}} \int_{B_{n}} f d v_{x^{\prime}}\right|_{\mathcal{R} \cap B_{n}} \\
& \leq \sup _{n} \frac{1}{\max \left\{1, \mu_{n}\left(B_{n}\right)\right\}}\|f\|_{L_{w}^{1}\left(v_{U}\right)}=\sup _{n} \frac{1}{\max \left\{1, \mu_{n}\left(B_{n}\right)\right\}}\|f\|_{v}<+\infty,
\end{aligned}
$$

where the last equality is due to the isometry between $L_{w}^{1}(v)$ and $P_{L_{w}^{1}(v)}(U)$. Consequently we also have that $f_{n} \in L^{1}\left(\mu_{U}\right)$ for every $n$, since $L^{1}\left(\mu_{U}\right)$ is an ideal of measurable functions with $f_{n} \uparrow f \mu_{U}$-a.e. and $f \in L^{1}\left(\mu_{U}\right)$. The dominated convergence theorem gives now that $\left(f_{n}\right)_{n}$ actually converges to $f$ in the norm of $L^{1}\left(\mu_{U}\right)$.

On the other hand, following (iii), there is a regular method of summability $D$ such that $\left(f_{n}^{D}\right)_{n}$ converges $v$-a.e. to $f^{D} \in E$. However, by the definition of regular method of summability, $\left(f_{n}^{D}\right)_{n}$ also converges to $f$ in $L^{1}\left(\mu_{U}\right)$, so there is a subsequence $\left(f_{n_{k}}^{D}\right)_{k} \subset\left(f_{n}^{D}\right)_{n}$ converging $\mu_{U}$-a.e. to $f$ (see Theorem 100.6 in [20]) and so $v_{U}$-a.e. Hence, since $v$-a.e. convergence and $v_{U}$-a.e. convergence are equivalent in $E$, we obtain that $f^{D}=f$ in $E$ which gives a contradiction. 
Theorem 1.1 can be established for ideals of $L_{w}^{1}(v)$ by requiring an extra condition on the vector measure $v$ : the so-called locally $\sigma$-finiteness of the measure (see $[4,8]$ and the references therein). Let $\mathcal{R}$ be a $\delta$-ring of subsets of $\Omega, X$ a Banach space and $v: \mathcal{R} \rightarrow X$ a vector measure. We say that $v$ is locally $\sigma$-finite (with respect to $\mathcal{R}$ ) if given $B \in \mathcal{R}^{l o c}$ with $\|v\|(B)<+\infty$, it is possible to write $B$ as $B=\left(\bigcup_{n} A_{n}\right) \cup N$, where $\left(A_{n}\right)_{n} \subset \mathcal{R}$ and $N \in \mathcal{R}^{\text {loc }}$ is a $v$-null set. Let us show an easy example of such property.

Example 2.1. Take a non countable set of indexes I and consider the $\delta$-ring $\mathcal{R}_{I}$ of all the finite subsets of $I$. In this case, $\mathcal{R}_{I}^{\text {loc }}$ is given by all the subsets of $I$. Let $1 \leq p \leq \infty$. The map $v_{p}: \mathcal{R}_{I} \rightarrow \ell^{p}(I)$ given by $v_{p}(A):=\sum_{i \in A} e_{i}$ is a vector measure, where $e_{i}$ is written for the $i$-th canonical unit vector, $i \in I$. If $1 \leq p<\infty$, we clearly have that the only subsets $B \in \mathcal{R}_{I}^{\text {loc }}$ satisfying the condition $\left\|v_{p}\right\|(B)<+\infty$ are the ones in $\mathcal{R}_{I}$, and so the measure is trivially locally $\sigma$-finite. However, it is not $\sigma$-finite. Note also that for $p=\infty$, the measure is not locally $\sigma$-finite, since all the sets of $\mathcal{R}_{I}^{\text {loc }}$ satisfy that $\left\|v_{\infty}\right\|(B)<+\infty$ and the non countable ones cannot be covered by a countable family of finite sets (note that $\|v\|(B)=0$ if and only if $B=\varnothing)$.

The same arguments allow to extend this example to the case of non atomic measures having values in non countable $\ell^{p}(I)$-sums as $\oplus_{\ell^{p}(I)} L^{1}\left(\mu_{i}\right)$, where $\mu_{i}$ is a non atomic probability measure for each $i \in I$ (see Example 3.4 in [12]).

Although a lot of usual situations are covered by Theorem 1.1, the next Theorem 2.3 is relevant because it can be applied directly to spaces with the Fatou property, since they can be represented as spaces $L_{w}^{1}(v)$ of vector measures (see Theorem 10 in [8]). In general, it is not the case that we have a natural identification of a particular Banach lattice with the Fatou property as an ideal of a space $L^{1}(v)$. For showing this, discrete vector measures are enough. Let us show an example.

Example 2.2. Consider the countable version of the example above -i.e. $I=\mathbb{N}-$ for the case $p=\infty$. Obviously, in this case the measure $v_{\infty}$ is $\sigma$-finite (and so locally $\sigma$-finite). It can be easily checked that the space $L^{1}\left(v_{\infty}\right)$ for the measure $v_{\infty}$ contains $c_{0}$. However, it does not contain $\ell^{\infty}$ but a direct argument just using the definition of weakly integrable function shows that $\ell^{\infty}$ is contained in $L_{w}^{1}\left(v_{\infty}\right)$.

Thus, Theorem 1.1 cannot be applied to the ideal $\ell^{\infty}$. The following one can do the work in this case.

Theorem 2.3. Let $v$ be a Banach space valued locally $\sigma$-finite measure. Let $E$ be a Banach

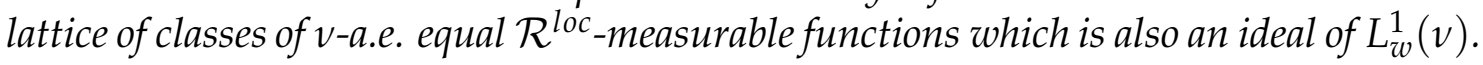
The following statements are equivalent.

(i) E has the weak $\sigma$-Fatou property.

(ii) E has the Komlós property.

(iii) For each norm bounded sequence $\left(f_{n}\right)_{n} \subset E$ there is a regular method of summability $D$ such that $\left(f_{n}^{D}\right)_{n}$ converges $v$-a.e. to a measurable function $f^{D}$ in $E$. 
Proof. Suppose that $v$ is locally $\sigma$-finite. Theorem 4.2 in [12] gives $(i) \Leftrightarrow$ (ii). A quick look to the proof of $(i i) \Rightarrow$ (iii) in 1.1 shows that it works also in this context. Finally, Theorem 4.8 in [4] assures the existence of a sequence of $\mathcal{R}$-simple functions which converges $v$-a.e. to $f$ for each $f$ in $L_{w}^{1}(v)$. Consequently, the arguments in the proof of $($ iii $) \Rightarrow(i)$ of Theorem 1.1 prove this implication again.

To finish this note, let us answer a question that appears in a natural way: is it true that all the Banach lattices of measurable functions with the weak $\sigma$-Fatou property satisfy the statements (ii) and (iii) of Theorem 2.3? Example 4.6 in [12] shows that this is not the case. If we consider the measure $v_{\infty}$ in Example 2.1, we have that $L_{w}^{1}\left(v_{\infty}\right)=\ell^{\infty}(I)$ (see Example 4.4 in [12]). This space is a Banach lattice with the weak $\sigma$-Fatou property. However, it is shown in Example 4.6 of [12] that this space has not the Komlós property, so without the local $\sigma$-finiteness assumption on the measure the equivalences given in Theorem 2.3 are not longer true.

\section{References}

[1] C.D. Aliprantis, A. Burkinshaw, Positive Operators, Springer, Dordrech, 2006.

[2] C. D. Aliprantis and K. C. Border, Infinite Dimensional Analysis, Springer, Berlin, 1999.

[3] J. K. Brooks and N. Dinculeanu, Strong additivity, absolute continuity and compactness in spaces of measures, J. Math. Anal. Appl. 45, 156-175 (1974).

[4] J.M. Calabuig, O. Delgado, M.A. Juan and E.A. Sánchez Pérez On the Banach lattice structure of $L_{w}^{1}$ of a vector measure on a $\delta$-ring Collectanea Mathematica, DOI 10.1007/s13348-013-0081-8 (2013).

[5] G. P. Curbera, El espacio de funciones integrables respecto de una medida vectorial, Ph. D. Thesis, Univ. of Sevilla, 1992.

[6] J.B. Day, C. Lennard, Convex Komlós sets in Banach function spaces, J. Math. Anal. Appl. 367, 129-136 (2010)

[7] O. Delgado, $L^{1}$ - spaces of vector measures defined on $\delta$-rings Arch. Math. 84, 432-443 (2005).

[8] O. Delgado and M. A. Juan, Representation of Banach lattices as $L_{w}^{1}$ spaces of a vector measure defined on a $\delta$-ring, Bull. Belg. Math. Soc. Simon Stevin Volume 19, Number 2, 239-256 (2012).

[9] C. L. DeVito, Functional Analysis, Pure and Applied Math. 81, Academic Press, New york, San Francisco, London, 1978.

[10] J. Diestel and J.J. Uhl, Vector Measures, Math. Surveys, vol. 15, Amer. Math. Soc., Providence, RI, 1977.

[11] P. Erdös and M. Magidor, A note on Regular Methods of Summability and the Banach-Saks Property, Proc. Amer. Math. Soc. 59, Number 2, 232-234 (1976). 
[12] E. Jiménez Fernández, M.A. Juan, E.A. Sánchez Pérez, A Komlós Theorem for abstract Banach lattices of measurable functions, J. Math. Anal. Appl. 383, 130136 (2011).

[13] D.R. Lewis, Integration with respect to vector measures, Pacific J. Math. 33, 294307 (1970).

[14] J. Lindenstrauss and L. Tzafriri, Classical Banach Spaces II, Springer, Berlin, 1979.

[15] S. Okada,W.J. Ricker, E.A. Sánchez Pérez, Optimal domain and integral extension of operators acting in function spaces. Operator Theory: Advances and Applications. 180. Birkhäuser Verlag, Basel, 2008.

[16] P.R. Masani and H. Niemi, The integration theory of Banach space valued measures and the Tonelli-Fubini theorems. I. Scalar-valued measures on $\delta$-rings, Adv. Math. 73, 204-241 (1989).

[17] P.R. Masani and H. Niemi, The integration theory of Banach space valued measures and the Tonelli-Fubini theorems. II. Pettis integration, Adv. Math. 75, 121167 (1989).

[18] G. F. Stefansson, $L^{1}$ of a vector measure Le Matematiche, Vol.XLVIII(1993)Fasc.II, pp.219-234.

[19] W.A.J.Luxemburg and A.C.Zaanen, Riesz Spaces I, Norht-Holland, Amsterdam, 1971.

[20] A.C.Zaanen, Riesz Spaces II, North-Holland, Amsterdam, 1983.

Departament d'Economía.

Facultat de Ciències Jurídiques y Econòmiques.

Universitat Jaime I.

Campus del Riu Sec

12071 Castelló de la Plana. Spain.

email :jimeneze@uji.es

Departamento de Contabilidad, Finanzas y Control de Gestión.

Facultad de Administración y Dirección de Empresas.

Universidad Católica de Valencia San Vicente Mártir.

C/Corona, 34. 46003 Valencia. Spain.

email: ma.juan@ucv.es

Instituto de Matemáticas Pura y Aplicada (IUMPA). Universitat Politècnica de València.

Camí de Vera $\mathrm{s} \backslash \mathrm{n}$.

46022 València. Spain.

email : easancpe@mat.upv.es 